Voix et Images

voixetimages

\title{
Bibliographie de Louky Bersianik
}

\section{Andrée De Rome}

Volume 17, numéro 1 (49), automne 1991

Louky Bersianik

URI : https://id.erudit.org/iderudit/200944ar

DOI : https://doi.org/10.7202/200944ar

Aller au sommaire du numéro

Éditeur(s)

Université du Québec à Montréal

ISSN

0318-9201 (imprimé)

1705-933X (numérique)

Découvrir la revue

Citer ce document

De Rome, A. (1991). Bibliographie de Louky Bersianik. Voix et Images, 17(1),

75-98. https://doi.org/10.7202/200944ar d'utilisation que vous pouvez consulter en ligne.

https://apropos.erudit.org/fr/usagers/politique-dutilisation/ 


\title{
Bibliographie de Louky Bersianik
}

\author{
par Andrée De Rome, \\ Université du Québec à Montréal
}

\section{MONOGRAPHIES}

L'Euguélionne, roman triptyque, Montréal, la Presse, 1976, 399 p. Photographie de couverture et dessins de Jean Letarte. Rééditions: Paris, Hachette-Littérature, 1978, 399 p. Photographie de couverture de Jean Letarte; les Éditions Alain Stanké, 1985, 412 p. (Québec 10/10). Dossier comprenant des extraits de la critique et un texte inédit de l'auteure: *Un roman qui n'en est pas un *. Mlustration de couverture de Suzanne Brind'Amour à partir d'une statuette d'argent de Jean Letarte.

La Page de garde (poème), St-Jacques-le-Mineur, Éditions de la Maison, 1978, [n.p.]. Embossage de Lucie Laporte, emboîtage de Pierre Ouvrard.

Le Pique-nique sur l'Acropole. Cahiers d'Ancyl (fiction $\varphi$ et $\psi$ ), Montréal, V.LB éditeur, 1979, 243 p. Eaux-fortes et tailles douces de Jean Letarte.

Maternative. Les Pré-Ancyl (textes poétiques et dramatiques), Montréal, VLB éditeur, 1980, 169 p. Acides de Jean Letarte.

Les Agénésies du vieux monde (essai), Outremont, l'Intégrale éditrice, 1982, 24 p. Reproduit dans la Main tranchante du symbole.

Au Beau milieu de moi (poèmes), Montréal, Éditions Nouvelle Optique, 1983, 85 p. Photographies de Kèro, cosignataire.

Axes et eau, poèmes de "la Bonne Chanson", Montréal, VLB éditeur, 1984, 231 p. Dessins de Francine Simonin.

Kerameikos (poèmes), Saint-Lambert, Éditions du Noroît, 1987, [n.p.]. 72 dessins de Graham Cantieni, cosignataire.

La Main tranchante du symbole (textes et essais féministes), Montréal, Éditions du Remue-ménage, $1990,280 \mathrm{p}$.

\section{CONTRIBUTIONS}

\section{II.1 Livres}

*Avertine la folle alliée (monologue), Te prends-tu pour une folle, madame Chose?, Montréal, Éditions de la Pleine Lune, 1978, p. 21-24.

"Le corps indigène " (nouvelle), Célibataire pourquoi pas?, Québec, Éditions Serge Fleury, 1981. 
"Je suis la ménade...*, Au fond des yeux. 25 Québécoises écrivent, Montréal, Nouvelle Optique, 1981, 109 pages, p. 10-13. Photographies de Kẻro. Reproduit dans la Main tranchante du symbole.

"Ouvrage de dame (essai), les CEuvres de création et le français au Québec. Actes du congrès Langue et Société au Québec, Tome III (1982), Editeur officiel du Québec, 1984, p. 219-230. Textes colligés et présentés par Irène Belleau et Gilles Dorion.

*En marge d'un roman qui n'en est pas un *, l'Euguélionne, Montréal, les Éditions Alain Stanké, 1985, p. 403-408 (Québec 10/10).

*Arbre de pertinence et utopie (essai), l'Emergence d'une culture au féminin, Montréal, Éditions Saint-Martin, 1987, p. 117-132 (Femmes). Actes du colloque international «Émergence d'une culture au féminin », Université de Montréal, avril 1982. Sous la direction de Marisa Zavalloni. Reproduit dans la Main tranchante du symbole.

«À première vue » (fiction), Premier Amour, Montréal, Éditions Stanké, 1988, p. 59-65 (Québec 10/10).

* La lanterne d'Aristote, essai sur la critique", la Théorie un dimanche, Montréal, les Éditions du Remue-ménage, 1988, 208 pages, p. 79-106. Cosignataires du livre: Nicole Brossard, Louise Cotnoir, Louise Dupré, Gail Scott et France Théoret. Reproduit dans la Main tranchante du symbole.

"Le portique des noms propres" (fiction), la Théorie un dimanche, op. cit., p. 107-115.

*Le lieu privilégié de l'attentat* (poème), Polytechnique 6 décembre, Montréal, les Éditions du Remue-ménage, 1990, p. 15-22. Sous la direction de Louise Malette. Également paru dans le Littéraire de Laval.

«Quadrille sanglant „, Polytechnique 6 décembre, op. cit., p. 45-50. Reproduit dans la Main tranchante du symbole.

«La femme et l'Hexagone», dans la Poésie de l'Hexagone, Montréal, l'Hexagone, 1990, p. 79-85.

\section{II.1.i À paraître}

«Les corps criblés de trous" (dernier chapitre de l'Euguélionne), dans Suzanne Lamy et al., Surréalisme et modernité au Québec, Paris.

\section{II.2 Périodiques}

II.2.1 Fiction et poésie

«Smaragdin * (poème), Rythmes et couleurs, n 18, Paris, juillet-août 1960, p. 33-36. Signé Lucile Durand; trame poétique du film d'animation Smaragdin de Jean Letarte, Paris, 1960.

«L'arbre à trous» (nouvelle); Châtelaine, décembre 1964. Signée Lucile Durand.

« me tangere * (poèmes), la Barre du jour, n 56-57, mai-août 1977: «Le corps, les mots, l'imaginaire ", p. 148-164. 
«Linstantané " (poèmes), la Nouvelle Barre du jour, n ${ }^{\infty}$ 68-69, septembre 1978: «Faits divers «, p. 78-82. Mlustrations de Jean Letarte.

*Le jazz des nébuleuses * (petite pièce à deux personnages), Sorcières, $n^{\circ} 14$, Paris, Stock, 1978: «La jasette ». Mlustration de Louisa Nicol.

"L'amour lesbien est une splendeur* (poèmes), la Nouvelle Barre du jour, $n^{\circ}$ 75, février 1979: «Célébrations », p. 65-74.

*La dérive du continent noir *, le Devoir, 24 novembre 1979: «Pour limaginaire *.

«La maternité mâle» (extrait du Pique-nique sur l'Acropole), Etudes littéraires, décembre 1979: «Le féminaire *, p. 407-410.

«La noyée repentie: fiction cinématographique* (nouvelle), Journal of Canadian Fiction, n* 25-26, 1979: «Les romanciers québécois et leurs cuvres", p. 68-75.

«Les paramécies massacrées* (extrait de l'Euguélionne), Canadian Women's Studies / les Cahiers de la femme, vol. I, $\mathrm{n}^{\circ} 3$, 1979: «Creativity Celebrating Us/La créativité pour nous célébrer *, p. 74-75.

* Scramville * (prologue, extrait du roman inédit le Squonk), la P'tite Presse littéraire, magazine d'information de VLB éditeur, vol: $\mathrm{I}, \mathrm{n}^{\circ} 3$, décembre 1979. Cahier spécial à l'occasion du Salon du Livre de Montréal.

"Séjournale. Maternative deux" (poèmes), Canadian Women's Studies / les Cahiers de la femme, vol. I, $\mathrm{n}^{\circ}$ 3, 1979: “Creativity Celebrating Us/La créativité pour nous célébrer ", p. 99-102.

*L'union fondamentale. Maternative trois* (poèmes), Possibles, vol. IV, n 1 , Mọntréal, automne 1979: «Des femmes et des luttes », p. 61-66.

«Le corps indigent * (poèmes), la Nouvelle Barre du jour, n* 92-93, juin 1980: «Pósie 80 \%, p. 33-38.

*La natte et la huttex (petite pièce à deux personnages), la Nouvelle Barre du jour, $\mathrm{n}^{\circ}$ 87, 1980, «La Mermour », p. 31-47.

*(A la) merci pour tout » (nouvelle), Féminin pluriel, vol. I, n 2, octobre 1981, p. 32-36. Illustration de Anne-Louise Duranleau.

*Les heures * (nouvelle), la Nouvelle Barre du jour, $\mathrm{n}^{\circ}$ 106, octobre 1981: *Les femmes et l'humour *, p.23-36.

«Sainte-Urbaine-la-Villaine» (poèmes); la Nouvelle Barre du jour, $\mathrm{n}^{\circ}$ 102, avril 1981: «La femme et la ville», p. 69-75.

«Corps de noce corps de divorce. Extrait de la Bonne Chanson, 2- Chanson pour Aphélie au beau regard " (première version), Résistances, $\mathrm{n}^{\mathbf{0}} \mathbf{3 - 4}$, printemps 1983: «Culture et lutte des femmes », p. 41-45. Ilustration de Philippe Sorel. Version définitive (revue et augmentée), dans Canadian Literature, $n^{\circ} 100$, printemps 1984: « 25th Anniversary Issue », p. 24-29.

*Mon dictionnaire des noms propres, *-A-Adizetu, suivi de la Bonne Chanson *, «Supplément au dictionnaire *, *Chanson pour Adizetu aux yeux luisants", «Les héros de la circoncision *, la Nouvelle Barre du jour, n* 122-123, février 1983: «Écritures ", p. 34-39. Photographie de Jania Mac Gillivray. 
*Sosie * (nouvelle), la Vie en rase, $n^{\circ}$ 12, juillet 1983: * Spécial été *, p. 21-24.

* Mon Dictionnaire des noms propres (suite): -A- Ahinsa, suivi de la Bonne Chanson (extraits): chanson pour Ahinsa-qui-ourlait-des-proies (Corps de noce corps de négoce), la Nouvelle Barre du jour, $n^{\circ} 140$, juin 1984: *Sortie/Exit \#, p. 26-27.

«Nuque lé(g)ère, chanson pour Ancyl aux longs cils» (poème), Parallelogramme, vol. X, $\mathrm{n}^{\circ}$ 1, ANNPAC/RACA, Montréal/Toronto, automne 1984, p. 30 .

* Permafrost ou le Doigt de Dieu (extrait du roman inédit $D u$ beurre de plomb dans l'aile), 10-5155-20 Art contemporain / Contemporary Art, n 9-10, Sherbrooke, Éditions Alternatives; été 1984: «La mort», p. 34-38. Photographie de Graham Cantieni.

*Amaro/Paid» (poème, première version), Montreal Now, vol. II, n 2, 1985 [n. p:].

«Le jeu des genres», Canadian Women's Studies / les Cahiers de la femme, vol. VI, n 3, été-automne 1985, p. 67.

"Party ou partouse, petit essai-fiction pour un essai de "party (mixte)" ", la Nouvelle Barre du jour, $\mathrm{n}^{\circ}$ 150, mars 1985: *Party (mixte): 10 ans d'écriture féministe "; p. 10-15.

«Le plaisir de l'écriture », Arcade, n 9, février 1985: « Rêves et fantasmes », p. 7-9.

«Étéa , la Nouvelle Barre du jour, n* 189-190, 1986: *Installations/Fictions », [n. p.]. Ilustrations de Francine Simonin.

*Une histoire sans orthographe „, la Parole métèque, $n^{\circ} 2,1987$, p. 15. Aussi paru dans Travers, $\mathrm{n}^{\circ} 31-32$, juin 1987, p: 2-5.

* La chaussée des géantes», Dalhousie French Studies, vol. XIII, automne/ hiver 1987: «Simone de Beauvoir et les féminismes contemporains *. Aussi paru dans Trois, vol. V, n* 1-2, automne 1989: *Femmes international ", p. 117.

«Temp/Press * (poème), Tessera, vol. XI, n* 2-3, Winnipeg, printemps/été 1988 : «Contemporary Verse 2 », p. 89.

*Le lieu privilégié de l'attentat * (poème), le Littéraire de Laval, 'vol. VI, $\mathrm{n}^{\circ} 1$, 1990, p. 11-13. Également paru dans Polytechnique 6 décembre, p. 15-22.

\section{II.2.2 Essais}

* Claude Gauvreau. Sur fil métamorphose» (chronique littéraire), le Devoir, 17 novembre 1956, p. 5-6. Signé Lucile Durand.

«Blocus des naissances vs Blocus de Berlin * (texte d'actualité politique), Cité libre, décembre 1961, p. 5-7. Signé Lucile Durand.

*A propos d'une déesse sumérienne: Lucie Laporte* (texte critique sur les cuvres picturales de L. Laporte), Vie des Arts, vol. XXII, n' 93, hiver 1978-1979: *À la recherche du Saguenay/Lac-St-Jean *, p. 54-57. Photographies de Gilles Dempsey. 
*Accoucheur ou marchand d'esclaves* (texte critique sur les éditeurs), le Devoir, novembre 1979. Cahier spécial à l'occasion du Salon du Livre de Montréal.

*Mon engagement féministe *, Canadian Women's Studies / les Cahiers de la femme, vol. II, $\mathrm{n}^{\circ}$ 4, 1980: «Law and Politics/La loi et la politique», p. 14. Photographie de Diana Davis. Reproduit dans la Main tranchante du symbole.

*Tradition féminine en littérature ", Revue de l'Université d'Ottawa / University of Ottawa Quartely, vol. $\mathrm{L}, \mathrm{n}^{\circ} 1,1980$ : «Conférence des femmesécrivains en Amérique et Conférence Vanier *, p. 24-27.

*Fieffée désirante \#, la Nouvelle Barre du jour, $\mathrm{n}^{\star}$ 118-119, novembre 1982: "Traces, écriture de Nicole Brossard, colloque NBJ 1982 ", p. 99-112.

"Pourquoi j’écris", Québec français, n 47, 1982: "Femmes et écritures", p. 30. Reproduit dans la Main tranchante du symbole sous le titre « J'ai cris ".

«La femme, l'ennemie à abattre. La vidéo pornographique", la Presse, 15 juin 1983.

¿Feu vert à l'escalade de la violence. La loi sur le cinéma et la pornographie ", le Soleil, 21 juin 1983.

«Lâchez les fauves " (texte sur la loi 109), le Devoir, 2 juillet 1983.

*Les réseaux de l'écriture * (proposition d'un atelier d'écriture), Arcade, $\mathrm{n}^{\star}$ 4-5, septembre 1983: «Actes du colloque au Cégep de Rosemont: Création et enseignement", p.113-133.

"L'herbe était rouge et comme rôtie... (La fiction introuvable) \#, la Nouvelle Barre du jour, $\mathrm{n}^{\circ} 141$, septembre 1984: «Vouloir la fiction, () la modernité», p. 47-48. Deuxième colloque bissextile, 29 février 1984. Reproduit dans la Main tranchante du symbole.

*Y 2-Q 2-B 2-CL *, Parallelogramme, vol X, n॰ 1, ANNPAC/RACA, Montréal/ Toronto, automne 1984, p. 31-32. Traduction de R. McGee, p. 17-18 et reproduit dans Women's Peace Write / Rites des femmes pour la paix et dans Canadian Women and Peace, p. 231-235.

*L'Autrement pareille, de Marguerite Andersen *, Arcade, n 10, octobre 1985, p. 52-53.

"L'espace encombré de la signature ", Tessera / la Nouvelle Barre du jour, $\mathrm{n}^{\circ}$ 157, septembre 1985: «L'écriture comme lecture », p. 91-97. Reproduit dans la Main tranchante du symbole.

"La mémoire courte i, la Vie en rose, juillet-août 1985; p. 50.

*Ô Nuit qu'en est-il de la transparence? \#, Estuaire, n* 40-41, 1985: «L'art poétique *, p. 23-27.

*Tout ça c'est du cinéma *, Copie Zéro, revue de cinéma, no 23 , Cinémathèque québécoise/Musée du cinéma, février 1985: "Anne-Claire Poirier", p. 33-34. 
* Comment naître femme sans le devenir », la Nouvelle Barre du jour, $\mathrm{n}^{\circ} 172$, mars 1986: «Forum des femmes, 21 avril 1985, YWCA», p. 57-65. Reproduit dans la Main tranchante du symbole.

*L'empire du statu quo», le Devoir, 9 août 1986. Aussi paru dans Tessera, vol. XI, n* 2-3, printemps /été 1988: *Contemporary.Verse 2», p. 42-47.

«L'éprouvette porteuse », le Devoir, 18 novembre 1987.

"Langue étrangère en quête d'auteure ", Possibles, vol. XI, n 3 , printempsété 1987: *Langue culture», p. 67-80. Reproduit dans la Main tranchante du symbole.

«Pré-liminaire», la Nouvelle Barre du jour, n 196, mars 1987: «Femmes scandales 1965-1985 », p. 67-80.

*Note pour un alphabet des révélations *, Tessera, vol. XI, $\mathrm{n}^{\circ} 5$, septembre 1988, p. 32-33. Reproduit dans la Main tranchante du symbole. Paru aussi dans le Littéraire de Laval, vol. VI, n 1, 1990, p. 21.

*Protestation gynile ", Possibles, vol. XIII, n* 4, automne 1989, p. 81-94. Reproduit dans la Main tranchante du symbole, texte revu et augmenté.

\section{II.3. Autres contributions}

\section{II.3.1 Anthologies}

*Ortygie l'inapparente. Maternative Une* (poèmes), Femme plurielle, Ottawa, université Carleton, 1979: * La poésie féminine et féministe québécoise contemporaine ", p. 21-24. Notice bio-bibliographique et photographie de l'auteure.

*Eremo* (extrait du roman inédit $D u$ beurre de plomb dans l'aile), Women and Words, The Anthology / les Femmes 'et les mots. Une anthologie, Vancouver, Harbour Publishing Co., 1984.

«Les fonctionnaires du phallus» (extraits du Pique-nique sur l'Acropole), dans Essais québécois, 1837-1983. Anthologie littéraire, par Laurent Mailhot et Benoît Melançon; Montréal; Hurtubise HMH, 1984, p. 463468 (Cahiers du Québec, Collection Textes et Documents littéraires).

* Nucléa épiphane „, la Pósie québécoise contemporaine, anthologie présentée par Jean Royer, Montréal/Paris, l'Hexagone/la Découverte, 1987, p. 198200. Déjà paru dans $A$ u beau milieu de moi.

«Party ou partouse, petit essai-fiction pour un essai de "party (mixte)" (extraits), la Barre du jour, la Nouvelle Barre du jour. Fin, p. 469-471.

*La splendeur " (extrait de Maternative), «Les yeux», «L'amour», «Cinquième mur * (extrait de Axes et eaux), Kerameikos (extraits), dans Nicole Brossard et Lisette Girouard, Anthologie de la poésie des femmes au Québec, Montréal, Éditions du Remue-ménage, 1991, p. 143-147.

\section{II.3.2. Publications diverses}

«La féminité, mot tendancieux (essai), postface du Calendrier du RemueMénage 1979, Montréal, 1978, quatrième de couverture. 
Extrait de l'Euguélionne, 1* Salon du livre, 23-27 mai, Paris, Grand Palais, 1981, p. 193.

Extraits de l'Euguélionne, chansons pour la paix, volume 1, Montréal, Passeport pour la paix (non daté), p. 7, 25, 45.

*Ailes arrivent» (pò̀me), Calendrier diu Remue-ménage 1982, mois de janvier, Montréal, 1981. Ilustrations de Louisette Gauthier-Mitchell.

* Les somnambules » (poèmes extraits de Maternative et extraits du Piquenique sur l'Acropole), l'Agenda du Remue-ménage 1983, textes du mois de juin, Montréal, Éditions du Remue-ménage, 1982.

*Corps de noce corps de divorce, Extrait de la Bonne Chanson, 2- Chanson pour Aphélie au beau regard * (première version), dans CalendrierAgenda 1984 / Saguenay Lac Saint-Jean, Résistances Éditeur, 1983.

*Frange dé-mesure» (poèmes pour l'événement «Réseau Art Femme»), Traces, Québec, Art Femme, catalogue artistique, Québec, 1983. Photographies de Louise Bilodeau.

*Temp/Press» (poème), dans 1984, Poetry Agenda Poésie, semaine du 28 mai, Sainte-Anne de Bellevue, The Muses' Company/La Compagnie des Muses, 1983. Reproduit dans Tessera, vol. XI, $\mathrm{n}^{*}$ 2-3, printemps-été 1988: «Contemporary Verse 2», p. 89. traduction de Erika Grundman. Titre original: $\propto$ Temp/Press $*$.

"Amaro/Paid» (poème, première version), dans World Poetry Festival, Festival Programme, Toronto, Harbourfront, 5 au 10 mai 1986. The League of Canadian Poets \& the Harbourfront Reading Series. Deuxième version, Poetry Canada Review, The League of Canadian Poets, World Poetry Festival of Toronto, mai 1986. La Boîte à poésie. La Première anthologie de poésie contemporaine du Canada sur ordinateur, Vancouver, Expo 1986, Pavillon du Canada.

*Y 2-Q 2-B 2-CL ", dans Women's Peace Write / Rite des femmes pour la paix, campagne de textes pour les députés, présentée sous forme de calendrier, Vancouver, Ed. West Coast Women and Word's Society, juin 1985-mai 1986. Déjà paru dans Parállelogramme, vol. $\mathrm{X}, \mathrm{n}^{\circ} 1, \mathrm{p} .31-32$ et reproduit dans «Up and Doing *, Canadian Women and Peace, p. 231-235 (traduction de R. McGee).

«Liber en feuille * (poème), dans La poésie est le cri de celui qui se fait, Hull, le Salon du Livre de l'Outaouais, * La nuit internationale de poésie, 22 mars 1986 ", [n.p.].

\section{II.3.3 Disque}

«Ballade à donner », «Le bout du fil ", « Chanson pour durer toujours", «Lá danse du monde ", «Oh Mayou ", «Sul'bord d'a track *, "Tit-Homme» (paroles de chansons), Trace et contraste, de Richard Séguin, Montréal, les Éditions de la roche éclatée (Capac), 1980. Prix à Spa et prix à Antibes, en 1981. 


\section{II.3.4 Cinéma}

Louky Doodle, de Jean Letarte, Paris, 1959. Co-scénarisation.

Smaragdin, de Jean Letarte, Paris, 1960. Texte poétique.

Carnaval du bout du monde, Prod. Marc Ellefsen, Chicoutimi, 1961. Texte narratif.

Un Vénutien en soucoupe volante, Claude Savard Cinéaste Inc. et la Caisse de dépôt du Québec, Montréal, 1970. Synopsis, scénario et dialogues.

Lettre de Léonard à Lüdovico il Moro, Claude Savard Cinéaste Inc. et Terre des Hommes, Montréal, 1970. Découpage dramatique, traduction et adaptation des textes.

La Terre de Cä̈n, Claude Savard Cinéaste Inc. et Hydro-Québec, Montréal, 1972. Texte narratif.

Le Jardin de Virulysse, les Films Gilbert Gratton de Montréal, Montréal, 1972. Synopsis, scénario et dialogues.

Alice et les femmes cinéastes, de Pierre Duceppe, Radio-Canada, Montréal, 1973. Recherche; documentation et texte de présentation.

\section{II.3.5. Radio et télévision}

Divers textes diffusés aux émissions suivantes de la radio de Radio-Canada:

Partage du matin.

\section{Méditation}

Pensée du matin

Pensée de la nuit

Nouveautés dramatiques

Une demi-heure avec

La Boîte aux merveilles ( 45 contes de 30 min., 1957-60)

Alternances

Féminisation du langage (20 demi-heures, FM)

Etc.

Textes diffusés à la télévision:

La Boutique de monsieur Nicola (CFTM, 1960-1962)

Chez Hélène (CBC, 1962-69)

La Boîte à surprise

Dictionnaire-magazine

Femmes d'aujourd'hui

Etc. 


\section{II.3.6 Théâtre et monologue}

Les Thesmophories d'Aristophane, ou Un espion à l'assemblée des femmes, adaptation libre de Louky Bersianik, pièce jouée à l'UQAM, décembre 1985: Mise en scène de Luce Guilbeault.

Une histoire sans orthographe, texte dit par Johanne Fontaine au Théâtre expérimental des femmes, dans le cadre du $4^{\circ}$ Festival de Création de Femmes: «L'érotisme „, Montréal, 1986.

Je vous salue Marie, mère porteuse, monologue dit par l'auteure, Foire internationale du livre féministe, Montréal, 1988. Aussi présenté aux Cinquante Heüres du féminisme, dans le cadre du cinquantième anniversaire du droit de vote des femmes, Montréal, avril 1990.

\section{PUBLICATIONS POUR ENFANTS *}

Koumic le petit esquimau, Montréal, les Éditions du Centre de Psychologie et de Pédagogie de Montréal, 1964. Ilustrations de Jean Letarte.

Le Cordonnier Pamphile mille-pattes, Montréal, les Éditions du Centre dé Psychologie et de Pédagogie de Montréal, 1964. Ilustrations de Jean Letarte.

La Montagne et l'escargot, Montréal, les Éditions dủ Centre de Psychologie et de Pédagogie de Montréal, 1965. Mlustrations de Jean Letarte.

Togo apprenti-remorqueur, Montréal, Les Éditions du Centre de Psychologie et de Pédagogie de Montréal, 1966. Mlustrations de Jean Letarte. Prix de la Province, 1966.

*La licorne», Crapauds et autres animaux, Montréál, la Courte Échelle, 1981.

Une vingtaine d'autres contes ont été publiés aux Éditions McGraw Hill Co. (1965-1970), et quelques extraits dans le Français vivant, lectures choisies, Montréal/Paris, Éditions françaises/Librairie Larousse, 1971.

\section{TRADUCTIONS}

\section{IV.1 Monographie}

The Euguelionne, triptych novel, Victoria/Toronto, Porcépic Press, 1982, 347 p. Traduction par Gerry Denis, Alison Hewitt, Donna Murray et Martha O’Brien. Introduction de.Jennifer Wealti-Walters.

\section{IV.2 Contributions}

\section{IV.2.1 Livres}

*Women's Work* (extraits d'un essai), dans In the Feminine: Women and Words / les Femmes et les mots, Conference Proceedings 1983, Chap. V: *Writing in the Feminine: Language and Form *, Edmonton, Longspoon Press, 1985, p. 155-165. Traduction: Erika Grundman. Titre original: "Ouvrage de dame .

* Tous ces textes sont signés Lucile Durand. 
*Y 2-Q 2-B 2-CL» (essai), Up and Doing, Canadian Women and Peace, Toronto, The Women's Press, 1989, p. 231-235. Édité par Janice Williamson et Deborah Gorham. Traduction: R. McGee.

*Aristotle's Lantern " (essai), A Mazing Space: Writing Canadian / Women Writing, Edmonton, Longspoon/NeWest Press, 1987, p. 39-48. Édité par Shirley Newman et Smaro Kamboureli. Traduction: A. J. Holden Verburg. Titre original : «La lanterne d'Aristote».

\section{TV.2.2 Périodiques}

* Drá me tangere " (poèmes, extraits de Maternative), Room of One's Own, vol. IV, n* 1-2, Vancouver, 1978, p. 98-100. Traduction: Barbara Godard. Parus aussi dans Northern Lit Quartely, vol. LXXXVIII, $\mathrm{n}^{\circ} 52$, Minneapolis, automne 1988, p. 13-17. Traduction: Barbara Godard et Érin Mouré.

*The Massacred Parameciae (extrait de l'Euguélionne), Canadian Women's Studies / les Cahiers de la femme, vol. I, n 3, Toronto/Montréal, 1979: * Creativity Celebrating Us/La Créativité pour nous célébrer », p. 74-75. Traduction: Wendy Johnston, Tima Newman, Howard Scott et Maureen Sullivan. Titre original: «Les paramécies massacrées *.

*To "Break the Sequence". Inscribe oneself in the Memory of the Future", Working Papers Series, $n^{\circ}$ 93, Wellesley (Mass., É.-U.), 1982: *International Symposium of Women Writers ". Traduction: Tamah Terry et Vicki Mistacco. Titre original : « Rompre la séquence * [inédit].

*New Cl(ear) Era. Song for Ancyl of the Long Lashes" (poème), Parallelogramme, vol. X, $n^{\circ} 1$, ANNPAC/RACA, Montréal/Toronto, automne 1984, p. 16. Traduction: R. McGee. Titre original: «Nuque Lé(g)ère, Chanson pour Ancyl aux longs cils». Extrait de Axes et eau, poèmes de "la Bonne Chanson » et paru dans Parallelogramme, vol. X, n' 1, 1984, p. 30.

*Y 2-Q 2-B 2-CL *, Parallelogramme, vol. X, n 1, Toronto/Montréal, automne 1984, p. 17-18. Traduction: R. McGee.

*Agenesias of the Old World » (essay), Trivia, $n^{\circ}$ 7, N. Amherst, MA, été 1985, p. 33-47. Traduction: Miranda Hay et Lise Weil. Titre original: les Agénésies du vieux monde.

*Foreign Language in Quest of an Author (essai), Raddle Moon, 1987. Traduction: Erika Grundman. Titre original: "Langue étrangère en quête d'auteur ».

*Sub/Urban-S(he)ain't Urban(e)* (poème), Raddle Moon 4, 1987, p. 14-17. Traduction: Erika Grundman. Titre original: *Sainte-Urbaine-laVillaine».

«Temp/Press. Song for Long-legged Epsilonne* (poème), Tessera, vol. XI, n* 2-3, printemps/été 1988: *Contemporary Verse $2 \%$, p. 91. Traduction: Erika Grundman. Titre original : *Temp/Press *.

"The Empire of the status quo. On The Decline of the American Empire", Tessera, vol. XI, n* 2-3, printemps/été 1988: «Contemporary Verse 2*, p. 48-53. Traduction: Erika Grundman. Titre original: "L'empire du statu quo $*$. 


\section{ENTRETIENS}

\section{V.1. Livre}

*Lucile Durand par elle-même *, dans Auteurs canadiens pour la jeunesse, 20 biographies et bibliographies, volume 1 , Montréal, CommunicationJeunesse, 1972 [n. p.].

"Louky Bersianik. Notre corps d'écriture ", dans Ecrivains contemporains. Entretiens I, 1976-1979 (recueil d'entretiens d'abord publiés dans le Devoir), Montréal, l'Hexagone, 1982. Propos recueillis par Jean Royer. Photographie de Kèro.

\section{V1.1 À paraître}

«La passion d'écrire", la Passion d'écrire. Recueil d'entretiens avec des auteur-es québécois-es, Montréal, Stanké. Propos recueillis par Alain Stanké.

\section{V.2. Périodiques}

"Louky Bersianik: "l'égalité avant la réciprocité" ", la Presse, samedi 20 mars 1976. Propos recueillis par Anne Richer.

«Louky Bersianik, la femme du mois *, Femme, vol. III, n 10, octobre 1977, p. 63-65. Propos recueillis par Camille Larose. Photographies d'enfance.

-Louky Bersianik : "Un nom bien à moi que personne d'autre ne porte" ", la Tribune, samedi 29 décembre 1979. Propos recueillis par Pierrette Roy.

«Louky Bersianik, de la mythologie vers le “je”, "Portrait d'elle", Louky Bersianik. Un pique-nique inusité », Femme du Québec, vol. I, n ${ }^{\circ}$, Montréal, janvier-février 1980, p. 5-6. Propos recueillis par Lorraine Leclerc.

«Entretien avec Louky Bersianik», le Bulletin Pantoute, no 5, Québec, printemps 1981. Propos recueillis par Marc Chabot et Josée Chaput.

«Lucile Durand interviewe Louky Bersianik ", Lettres québécoises, $n^{\circ} 26$, juin 1982, p. 53-55. Photographie de Athé.

*Entrevue avec Louky Bersianik», Féminin pluriel, juin 1982. Propos recueillis par Gloria Escomel.

"Louky Bersianik, inconsolable...*, Perspectives (la Presse), janvier 1982. Propos recueillis par Monique Roy. Photographie de Kèro.

*Louky Bersianik et la mythologie du futur. De la théorie-fiction à l'émergence de la femme positive *, Lettres québécoises, $\mathrm{n}^{\circ} 27$, automne 1982, p. 60-69. Propos recueillis par Donald Smith. Photographiés de Athé.

*Écrire au féminin. Interview avec Denise Boucher, Madeleine Gagnon et Louky Bersianik » (dix questions sur l'écriture des femmes au Québec, posées par écrit en juillet 1983 à chacune des trois auteures, qui y a répondu par écrit), Québec Studies, n 2 , une publication du Northeast Council for Quebec Studies, Darmouth College, Hanover, É.-U. 1984, p. 125-142. Propos recueillis par Karen Gould. 
*Louky Bersianik. Les terribles vivantes », le Devoir, samedi 9 mars 1985. Propos recueillis par Jean Royer.

« Entrevue: Louky Bersianik. Entre la dictée de l'inconscient et le tremblement de la conscience *, propos recueillis par Claudine Bertrand et Josée Bonneville, revus et augmentés par Louky Bersianik; Arcade, n' 11, février 1986: « Écrire en atelier *, p. 65-74.

"Comme en langue étrangère *, la Vie en rose, Montréal, septembre 1986, p. 26-29. Propos de Louky Bersianik et Louise Harel au sujet de la féminisation du langage, recueillis par Françoise Guénette.

Les Bâtisseurs de dictionnaires, entrevue du 13 mars 1986, 8* émission: «Le dictionnaire au féminin», Montréal, Société Radio-Canada. Propos recueillis par Rober Racine, repris et résumés par Lise Harou, Terminogramme, bulletin d'infoterminologie et de linguistique, $n^{*} 37-38$, octobre 1986, p. 7-13.

*Les hommes comme les femmes sont victimes du patriarcat „, le Devoir, 5 décembre 1990. Propos recueillis par Jean Basile.

\section{V.3. Radio}

Le Thavail de la création, dans le cadre de la série radiophonique du même nom, Cahier $n^{\circ} 21$, Service des Transcriptions et des dérivés de la radio, Montréal, Maison de Radio-Canada, 17 février 1982. Propos recueillis par Marcel Bélanger.

Les Bâtisseurs de dictionnaires, entrevue du 13 mars 1986, 8* émission: *Le dictionnaire au féminin*, Montréal, Société Radio-Canada. Propos recueillis par Rober Racine, repris par Lise Harou.

\section{V.4 Film}

TODD-HÉNAUT, Dorothy, les Terribles vivantes / Firewords, Office National du Film, Montréal, 1986. Un long métrage et/ou trois courts métrages, sur Louky Bersianik, Nicole Brossard et Jovette Marchessault.

\section{V.5 En traduction}

*Louky Bersianik: Die Mythologie der Zukunft: Ein Gesprach mit Donald Smith *, Die Horen: Zeitschrift fur Literatur, Kunst und Kritik 141, vol. 31, $\mathrm{n}^{\circ}$ 1, 1986. Propos recueillis par Donald Smith. Traduction allemande: Michael Mundhenk. Titre original: *Louky Bersianik et la mythologie du futur, une entrevue de Donald Smith». Déjà paru dans Lettres québécoises, $\mathrm{n}^{\circ}$ 27, automne 1982, p. 60-69.

\section{TEXTES RELATIFS A L'OUUVRE DE LOUKY BERSIANIK VI.1 Sujets variés ou généraux VI.1.1 Livres}

CLARK, Gerald, *Louky et Yvette „ Montréal, ses citoyens, son establishment, chap. III, Montréal, les Éditions de l'Homme, 1982, p. 43-59. 
Dictionary of Literary Biography, «Canadian Writers 1960. Second Series *, Detroit, Publication Yale Research, 1987, p. 11-16. Texte de Patricia Smart.

Dictionnaire des écrivains, Montréal, l'UNEQ, 1983.

DUCROCQ-PÓRIER, Madeleine, les Images littéraires des femmes québécoises. Les femmes québécoises depuis 1960, tome II: «En Littérature». Extrait d'une thèse de doctorat présentée à la Sorbonne et publiée par le C.N.R.S., Paris, 1979, 41 pages (grand format), p. 13, 14, 19 et 33.

GOULD, Karen, Writing in the Feminine: Feminism and Experimental Writing in Quebec (Etude portant sur Louky Bersianik, Nicole Brossard, Madeleine Gagnon et France Théoret), Carbondale, Ml., Southern Illinois University Press, 1990.

HAJDUKOWSKI-AHMED, Maroussia, «Louky Bersianik: Feminist Dialogisms", dans Traditionalism, Nationalism and Feminism: Women Writers of Quebec, Westport, CT, Greenwood, 1985, p. 205-225. Édité par Paula Gilbert Lewis et Elaine Marks.

NEUMAN, Shirley, «Importing Difference ", A Mazing Space: Writing Canadian Women Writing, Edmonton, Longspoon, 1986, p. 392-405. Édité par Shirley Newman' et Smaro Kamboureli.

PRÉVOST, Robert, Québécoise d'hier et d'aujourd'hui, Montréal, les Éditions Stanké, 1985.

WEALTI-WALTERS, Jennifer, * When Caryatids Move: Bersianik's view of Culture", A Mazing Space: Writing Canadian Women Writing, Edmonton, Longspoon, 1986, p. 298-306. Édité par Shirley Newman et Smaro Kamboureli.

\section{VI.1.2 Périodiques (excluant hebdos et quotidiens)}

BÉLANGER, Paul, «Nouvelle Barre du jour, l'écriture comme lecture (Tessera). *L'éspace encombré de la signature *, automne 1985, $n^{\circ} 157 *^{\prime}$; Nuit blanche, Essais québécois, commentaires, $\mathrm{n}^{\circ}$ 21, Montréal, décembre 1985/janvier 1986, p. 16.

CAMPEAU, Francine, «La Théorie, un dimanche, la Parole métèque, $\mathrm{n}^{\circ} 7$, automne 1988, p. 38.

COTNOIR, Louise, *Voyante aux prises ávec l'invisible *, Cahiers du symbolisme, à paraître automne 1991 .

FOURNIER, Danielle, «[...] la Théorie, un dimanche », Urgences, $\mathrm{n}^{\circ} 21$, novembre 1988, p. 106-108.

FRÉMONT, Gabrielle, *Petite histoire d'un grand mouvement. L'écriture des femmes", Questions de culture, $\mathrm{n}^{\circ}$ 9, Québec, Institut québécois de recherche sur la culture (IQRC), 1986: «Identités féminines: mémoire et création ", p. 173-182.

GOULD, Karen, « Setting words free: Feminist Writing in Quebec. Language as Colonizer \#, Signs, été 1981, p. 617-642. 
GOULD, Karen, *Female Tracings: Writing as Re-Vision in the Recent Works of Louky Bersianik, Madeleine Gagnon, and Nicole Brossard", History of Social Science Teacher, vol. XVII, n 2, hiver 1982, p. 73-82.

GOULD, Karen, *Spatial Poetics, Spatial Politics: Quebec Feminists on the City and the Countryside", American Review of Canadian. Studies, vol. XII, $\mathrm{n}^{\circ} 1$, été 1982 , p. 1-9.

HAMM, Jean-Jacques, «Présentation", Etudes littéraires, vol. XIX, $\mathrm{n}^{\circ} 1$, printemps-été 1986, p. 9.

LAROSE, Camille, «Louky Bersianik, la femme du mois", Femme, vol. III, $\mathrm{n}^{\circ} 10$, octobre 1977, p. 26-28.

MACKWARD, Christiane, «Quebec Women Writers», Women and Literature, vol. VII, $n^{\circ} 1,1977$.

MESSNER, Céline, «Les personnages mythologiques dans l'écriture des femmes ", Arcade, n 11, février 1986.

MONDOU, Pierrette, "À propos de "Frange, Dé-mesure" ", 10-5155-20 Art contemporain, $n^{\circ} 2$, automne 1982, p. 30 .

PICARD, Anne-Marie, «La Thérie un dimanche», University of Toronto Quartely, vol. LIX, n` 1, automne 1989, p. 206-209.

SAINT-MARTIN, Lori, «Les mots pour le redire ", Spirale, n 72 , septembre 1987, p. 18.

SAINT-MARTIN, Lori, «Écriture et combat féministe: figure de la sorcière dans l'écriture des femmes au Québec ", Québec Studies, printemps-été 1991, p. 67-82.

SIMON, Sherry, «La Théorie, un dimanche», Spirale, $\mathrm{n}^{\circ}$ 72, septembre 1987, p. 8.

SMART, Patricia, *Voices of Commitment and Discovery: Women Writers in Quebec ", Room of One's Own, vol. IV; n 1-2, 1978, p. 7-18.

SMART, Patricia, *Culture, revolution and politics in Quebec», The Canadian Forum, vol. LXII, n' 718, mai 1982, p. 7-10.

SMART, Patricia, «L'émergence d'une culture au féminin », Voix \& images, vol. XIII, $\mathrm{n}^{\circ} 1$, automne 1987, p. 158-161.

SUCHET, Simone, «Les Terribles vivantes ", Séquences, n 129, avril 1987, p. 60.

THÉRY, Chantal, « C'est dans la fiction au féminin que se joue le devenir du féminisme comme philosophie" (sur la Théorie, un dimanche), Lettres québécoises, $\mathrm{n}^{\circ} 51$, automne 1988, p. 49-50.

VOLDENG, Évelyne, «La poésie contemporaine d'inspiration féministe", Dérives, $\mathrm{n}^{\circ} 22 ; 1980$, p. 3-14.

VOLDENG, Évelyne, *Intertextualité dans les écrits féminins d'inspiration féministe ", Voix \& images, vol. VII, n 3 , printemps 1982, p. 523-530. 


\section{VI.1.3 Hebdos et quotidiens}

BOIVIN, Jean-Roch, *Ceci n'est pas une critique et la théorie a ses limites. La Théorie, un dimanchen, le Devoir, samedi 25 juin 1988.

CARON, Louis, *Louky Bersianik. Qui est-elle?», Courrier Sud, Nicolet, 4 mai 1976.

FORTIN, Marie-Claude, «La Théorie, un dimanche», Voir, septembre 1988.

MOREAU, Lise, «Louky Bersianik », la Presse, Astrologie, 24 mars 1976.

PARIZEAU, Alice, *Louky Bersianik: rage de femmes *, la Presse, 11 août 1986.

\section{VI.1.4 Film}

TODD-HÉNAUT, Dorothy, les Terribles Vivantes / Firewords, Office national du film, Studio D, Montréal, 1986. Un long métrage et/ou trois courts métrages, sur Louky Bersianik, Nicole Brossard et Jovette Marchessault.

\section{VI.2 L'Euguélionne}

\section{VI.2.1 Livres}

SCOTT, Howard, Voluntarist Action, Women's Language, Chap. IV et V, extraits de son Mémoire de Maîtrise es Arts: «Louky Bersianik's l'Euguélionne: Problems of Translating the Critique of Language in a New Quebec Feminist Writing ", Canadian Women Writing / les Ecrivaines canadiennes, A Shirley Newman's Bilingual Collection, Edmonton, NeWest Press, 1986 (Critical Documents).

VOLDENG, Évelyne, *La parodie carnavalesque dans l'Euguélionne (Bersianik) „, Féminité, subversion et écriture, Montréal, les Éditions du Remueménage, 1983, p. 119-126 (Itinéraires féministes).

WEALTI-WALTERS, Jennifer, *And Dwelt Among Us: The Euguelionne (Bersianik)m, chap. 8 dans Fairy Tales and the Female Imagination, Montréal et St. Albans (Vermont), Eden Press, 1982, p. 113-133.

WEALTI-WALTERS, Jennifer, *Introduction *, The Euguelionne, traduction, Victoria/Toronto, Porcépic Press, 1982, [n.p.].

\section{VI.2.2 Périodiques (excluant hebdos et quotidiens)}

AHMED, Maroussia, *Transgresser, c'est progresser: l'Euguélionne », Incidences, Université d'Ottawa, 1980, p. 119-127 (Romancières québécoises).

[ANONYME], «L'Euguélionne *, le Livre canadien, vol. VII, n 170, Montréal, mai 1976.

[ANONYME], «Des Québécois pas ordinaires», Nous, Montréal, janvier 1977, p. 57. Paru également dans The American Review of Canada Studies, vol. XIII, n², été 1983: «Québec aujourd’hui/ to-day », p.74-89.

CASTRO, Ginette (Université de Bordeaux III), «The Euguelionne ", Etudes canadiennes, $n^{\circ} \mathrm{XVI}, 1984$, p. 79-80. 
CLOUTIER, Cécile, *L'Euguélionne: texte et signification *, communication à la conférence des femmes-écrivains en Amérique, Revue de l'Université d'Ottawa, vol. L, n' 1, janvier-mars 1980, p. 95-98.

C.V., «L'Euguélionne », Québec français, mai 1976, p. 5.

DETREZ, Conrad, *L'Euguélionne (Hachette)», Magazine littéraire, $\mathrm{n}^{\circ}$ 138, juin 1978, p. 36.

FORŚYTH, Louise H., *L'écriture au féminin: l'Euguélionne de Louky Bersianik, l'Absent aigu, de Geneviève Amyot, l'Amer de Nicole Brossard *, Journal of Canadian Fiction, n* 25-26, 1979: «Les romanciers québécois et leurs œuvres *, p. 199-211.

FORSYTH, Louise H., *The Euguelionne: Out of this World», Broadside, vol. III, $n^{\circ} 6$, avril 1982.

FRANÇOISE, *Y en a qui appellent ça des livres, nous on appelle ça de l'énergie : l'Euguélionne », Mainmise, n³7, juin 1976.

GROULT, Benoite, *Alice et Zazie au Québec: l'Euguélionne de Louky Bersianik ", F Magazine, $\mathrm{n}^{\circ}$ 6, juin, 1978, p. 64-65.

L'HEUREUX, Christine, «L'Euguélionne », les Livres d'ici, n 33, 1976.

LEBRUN, Paule, «L'Euguélionne », Châtelaine, septembre 1976, p. 21.

LÉGER, Louise, «Revue du livre de Louky Bersianik: l'Euguélionne ", Libération, vol. VI, $\mathrm{n}^{\circ} 8(54)$, août-septembre 1976.

MARTIN, Agathe, «L'Euguélionne», Livres québécois, 1976.

MERIVALE, Patricia, *Idéologies, [...] The Euguelionne „, Canadian Literature, $\mathrm{n}^{\circ} 102$, automne 1984, p.130-134.

MESSNER, Céline, *Hommage à l'Euguélionne de Louky Bersianik», Tribune juive, vol. IV, $n^{\circ} 3$, novembre-décembre 1986, p. 24-25.

M.M., *Candide au féminin: l'Euguélionne», la Gueule ouverte, 12 juillet 1978.

RICARD, François, *L'Euguélionne *, Liberté, vol. XIII, n 105, mai-juin 1976, p. 91-94.

RICHARD, Robert-Gérald, «L'Euguélionne», Atlantis, A Women's Studies Journal, vol. II, $n^{\circ} 1$, automne 1976, p: 127-129.

SAINT-MARTIN, Lori, «L'ironie féministe prise au piège: l'exemple de $l ' E u$ guélionne, Voix \& images, vol. XVI, n 1, automne 1990, p. 110-121.

SPENCER, Kathleen L., *The Euguelionne, A triptych novel», Science Fiction \& Fantastic Book Review, nं 1, Vista; CA., mai 1982.

STANDEN, Bernice, *The Euguelionne, A triptych novel *, Canadian Book Review Annual, 1982, p. 138.

THÉRIO, Adrien, «Histoire pour Louky Bersianik (l'Euguélionne)», Lettres québécoises, $\mathrm{n}^{\circ} 2$, mai 1976, p.41.

TREMBLAY-GILLON, Micheline, *Le cri déserté. (Transportables de Jean Letarte et l'Euguélionne de Louky Bersianik)», Vie des Arts, vol. XXI, $n^{\circ}$ 85, hiver 1976-1977: * Art actuel », p. 74-75. 
URBAS, Jeannette, «The Euguelionne», Canadian Woman Studies, Vol. IV, $\mathrm{n}^{\circ}$ 2, hiver 1982, p. 93-94.

WILSON, Paul, «Life After Man. The Euguelionne», Books in Canada, février 1982.

\section{VI.2.3 Hebdos et quotidiens}

[ANONYME], «L'Euguélionne», les Enseignants, Revue d'information pédagogique, vol. VI, $n^{\circ} 7$, mars 1976, p. 11.

[ANONYME] «La Maudite Sauvagesse et l'Euguélionne s'attirent les éloges de la critique», Progrès-Dimanche, 19 décembre 1976.

BARCLAY, Pat, *French-Canadian Author Shows Women Have way to Go: The Euguelionne », Brandon Sun, Manitoba, 16 janvier 1982.

BARCLAY, Pat, «Quebec Novice's Rousing Call for Rights of Women:. The Euguelionne ", Victoria Times Colonist, 9 janvier 1982.

BARCLAY, Pat, * This Book Could Be the Surprise of the Season (le Monde): The Euguelionnew, Peterborough Examiner, 23 janvier 1982.

BASILE, Jean, "L'Euguélionne: la moitié des hommes sont une femme», le Devoir, samedi 6 mars 1976.

BEAUDET, Josée, «Le Québec à l'heure du féminisme, remue-ménage: l'Euguélionne", les Nouvelles littéraires, $\mathrm{n}^{\circ} 2547$, jeudi 26 août 1976.

BERNHEIM, Cathy, «La huitième famille de papier et quelques autres: à propos de l'Euguélionne », Libération, 6 juillet 1978.

CARON, Louis, «La mythologie féminine ou le monde à l'envers: l'Euguélionne», Courrier Sud, Nicolet, mardi 4 mai 1976.

CARVINGTON, Maureen, «The Dark Stain of Misogyny The Euguelionne», Monday Magazine, Victoria, B.C., 5-11 mars 1982.

CELLARD, Jacques, «Le Québec entre deux révoltes. Du côté des femmes: Une grande voix venue d'ailleurs: l'Euguélionne», le Monde, vendredi 4 novembre 1977.

CELLARD, Jacques, "Paroles de femmes: [...] l'Euguélionne», le Monde, Paris, 2 août 1976.

DEGARIE, Reine, *L'Euguélionne, toujours une source d'émerveillement *, le Droit, samedi 27 mai 1978.

FELX, Jocelyne, «L'Euguélionne », le Nouvelliste, mercredi 2 juin 1976.

GAGNON, Solange, "Que la femme vive enfin pour elle *, Montréal-Matin, 31 mai 1976.

HANS, Marie-Françoise, «L'Euguélionne de Louky Bersianik (Hachette) », les Nouvelles littéraires, 13-21 juillet 1982.

L'HEUREUX, Christine, «L'Euguélionne», la Voix gaspésienne, $\mathrm{n}^{\circ} 26$, mercredi 30 juin 1976.

LAFORGE, Christiane, «La préhistoire de la vie féminine: l'Euguélionne», le Quotidien du Saguenay-Lac-Saint-Jean, mercredi 17 novembre 1976. 
LAMY, Suzanne, «Livre étonnant aux Éditions la Presse: l'Euguélionne gargantuesque de Louky Bersianik *, le Jour, jeudi 26 février 1976.

LAROSE, Camille, *L'Euguélionnè *, Madame, juin 1976, p. 35.

MARTEL, Réginald, *Quand femme varie», la Presse, «L'Euguélionne de Louky Bersianik: vers le simple pouvoir d'être », samedi 20 mars 1976.

MARTEL, Réginald, *Avec lyrisme, avec humour... *, la Presse, 13 septembre 1976.

M.R., *Ainsi parlait l'Euguélionne ", les Têtes de pioche, Journal des femmes, $n^{\circ}$ 3, mai 1976

PAQUETTE, Diane T., «L'Euguélionne », l'Hebdo de Portneuf, vol. IV, n²4, lundi 14 juin 1976.

RAINVILLE, Andrée, «Une si réelle fiction! L'Euguélionne *, ProgrèsDimanche, 7 mars 1976.

RICHARD, Robert, «L'Euguélionne de Louky Bersianik: “Un homme sur deux est une femme " ; le Droit, samedi 5 juin 1976.

S.G., «Le livre (féministe), de l'été: l'Euguélionne», Journal dominical, Genève, 26 juillet 1976.

SCOTT, Gail, «Quebec's Latest Feminist Novel - From a Far-Out Perspective. L'Euguélionne », The Gazette, samedi 27 novembre 1976.

STEUR, W. R., *Quebec Satire Degenerates into a Polemic *, Winnipeg Free Press, 23 janvier 1982.

SUTHERLAND, Ronald, Two Works Mark the Explosion of Feminism in Quebec: [...] l'Euguélionne ", The Globe and Mail, dimanche, 21 décembre 1981.

TIMMERMAN, Darquise, *Louky" invente un contrat social fondé sur une égalité et une complémentarité des sexes », le Droit, 5 avril 1976.

TURGEON, Paule, «L'Euguélionne: quand la femme cherche sa planète positive", le Soleil, Les arts: "Louky Bersianik: un nouveau féminisme", samedi 20 mars 1976.

\section{VI.3 La Page de garde}

OUVRARD, Hélène, «Pòme/Gravure: Les Éditions de la Maison, [...] la Page de garde, Louky Bersianik/Lucie Laporte „, Cahiers des arts visuels au Québec, vol. III, n 9, Montréal, printemps 1981, p. 12-13.

\section{VI.4 Le Pique-nique sur l'Acropole}

\section{VI.4.1 Périodiques (excluant hebdos et quotidiens)}

ALONZO, Anne-Marie, «Le Pique-nique sur l'Acropole», la Gazette des femmes, avril 1980, p. 4.

[ANONYME], *L'auteur de l'Euguélionne sur campus. Elle maitrise le récit fantastique pour dialoguer crûment dans le présent „, Gazette, vol. XVII, $\mathrm{n}^{\circ} 1,1982$, p. $5-9$. 
CHABOT, Marc et CHAPUT, Sylvie, «Le festin des dieux ou de la difficulté de se mettre à table: le Pique-nique sur l'Acropole $»$ Discours d'ici, Vol. II, n 2, mai 1980, p. 116-137.

DAVID, Carole, «Louky Bersianik: un appel à la jouissance sur le corps majeur: le Pique-nique sur l'Acropole», la P'tite Presse littéraire, Magazine d'information de VLB éditeur, décembre 1979.

GREEN, Mary Jean, "Le Pique-nique sur l'Acropole», The French Review, vol. LIV, $\mathrm{n}^{\circ}$ 4, mars 1981, p. 615-616.

IMBERT, Patrick, *Débats sur les gynocides * (relecture du Pique-nique sur l'Acropole), Lettres québécoises, $\mathrm{n}^{\circ}$ 54, été 1989, p. 48-49.

LECLERC, Lorraine, «Louky Bersianik, de la mythologie vers le “JE”. Un pique-nique inusité », Femme du Québec, vol. 1, n 6, janvier-février 1980.

MARCOTTE, Gilles, «[...] Des dames entre elles „, l'Actualité, mars 1980, p. 80.

OUELLETTE-MICHALSKA, Madeleine, *Vie réelle et femme rêvée. Le Pique-nique sur l'Acropole », Châtelaine, mars 1980, p. 36.

PAYEUR-MINOT, Gaétane, «Le Pique-nique sur l'Acropole", Nos livres, novembre 1980 , p. 323-324.

THÉORET, France, *Entre l'excès du verbe et de la communication: le Pique-nique sur l'Acropole », Spirale, février 1980: «Fiction québécoise ».

VANASSE, André, «Du politique. Le Pique-nique sur l'Acropole», Lettres québécoises, $\mathrm{n}^{\circ} 17$, printemps 1980, p. 21-22.

VANDENDORPE, Christian, *Le Pique-nique sur l'Acropole», Québec français, mars 1980, p. 8.

\section{VI.4.2 Hebdos et quotidiens}

BLAIN, Daniẻle, «Quand les femmes jacassent... sur l'Acropole », le Journal de Montréal, samedi 19 janvier 1980.

HAMEL, Dorịs, «Pique-nique sur l'Acropole, une société sans soús-êtres humains », le Nóuvelliste, jeudi 6 décembre 1979.

MARTEL, Réginald, «Le Banquet s'achève et le Pique-nique devrait commencer», la Presse, samedi 22 décembre 1979.

OUELLETTE-MICHALSKA, Madeleine, «Louky Bersianik: un Pique-nique savoureux *, le Devoir, samedi 15 décembre 1979.

POULIN, André, «Pique-nique sur l'Acropole „, Matricule, Université Laval, Sainte-Foy, 21 octobre 1980.

ROYER, Jean, «Notre corps d'écriture (le Pique:nique sur l'Acropole)», le Devoir, Montréal, samedi 24 novembre 1979.

TREMBLAY, Régis, «Un Pique-nique où le sexe est servi froid \#, le Soleil, jeudi 17 janvier 1980. 


\section{VI.5 Maternative}

VI.5.1 Périodiques (excluant hebdos et quotidiens)

GREEN, Mary Jean, «Maternative. Les Pré-Ancyl „, French Review, vol. LV, $\mathrm{n}^{\circ} 4$, Mlinois, mars 1982, p. 563-564.

ROY, Monique, «La dernière maternité: Maternative», Livre d'ici, vol. VI, $n^{\circ} 23,11$ mars 1981.

\section{VI.5.2 Hebdos et quotidiens}

BEAULIEU, Ivanhoé, «Du politique à l'érotique: Maternative», la Presse, samedi 4 avril 1981.

OUELLETTE-MICHALSKA, Madeleine, «De la mère à la fille: Maternative», le Devoir, samedi 28 février 1981.

\section{VI.6 Les Agénésies du vieux monde}

VI.6.1 Périodique

ÉMOND, Ariane, «Les Agénésies du vieux monde [...]», la Vie en rose, marsavril-mai 1982, p. 66.

\section{VI.6.2 Quotidien}

ROYER, Jean, «La féminie intégrale: Notes pour une ontologie du féminisme radical, par Mary Daly \& les Agénésies du vieux monde, par Louky Bersianik : l'Intégrale éditrice », le Devoir, samedi 6 mars 1982.

VI.7 Au beau' milieu de moi

VI.7.1 Périodiques (excluant hebdos et quotidiens)

LAFRENIÈRE, Céline, « Kèro et Bersianik (Louky); Au beau milieu de moi ", Nos livres, vol. XIV, septembre 1983, p. 34.

LASSERRE, Claudette, "Tourner en rond: Au beau milieu de moi, photographies de Kèro, texte de Louky Bersianik», Spirale, $\mathrm{n}^{\circ} 38$, novembre 1983 (Photographie).

\section{VI.7.2 Hebdos et quotidiens}

RAYMOND, Claudette, * Kèro: une infinie tendresse: Au beau milieu de moi. Photographies de Kèro, texte de Louky Bersianik », le Devoir, samedi 25 juin 1983.

RICHER, Anne, «Au beau milieu de moi : un bonheur tout rond ", la Presse, samedi 11 juin 1983.

\section{VI.8 Axes et eau}

\section{VI.8.1 Périodiques (excluant hebdos et quotidiens)}

ALONZO, Anne-Marie, «Une autre dimension: Axes et eau ", la Vie en rose, $n^{\circ} 28$, juillet-août 1985, p. 55.

ANDERSEN, Marguerite, «Coureurs de bois et poètes féministes: [...] Axes et eau », Poetry Canada Review, vol. VI, n 4, été 1985, p. 16. 
BAYARD, Caroline, "Axes et eau, poèmes de "la Bonne Chanson" ", Lettres québécoises, $\mathrm{n}^{\circ} 39$, automne 1985, p. 53.

BROCHU, André, * Autour d'Alain Grandbois indélébile », Voix \& images, vol. $\mathrm{XI}$, n², hiver 1986, p. 341-342.

CHABOT, Marc, *Axes et eau ", Nuit blanche, hiver 1985, p. 9.

COTNOIR, Louise, «Axes et eau », Estuaire, n³5, printemps 1985, p. 92.

DÉSAUTELS, Denise, “Axes et eau, poèmes de "la Bonne Chanson" ", Arcade, n' 10 , octobre 1985, p. 53-54.

MESSNER, Céline, «Axes et eau», Canadian Woman Literature / les Cahiers de la femme, vol. VI, $\mathrm{n}^{\circ} 4$, hiver 1985, p. 127.

NEPVEU, Pierre, "L'essentiel et le frivole: Axes et eau", Spirale, $\mathrm{n}^{\circ} 52$, mai 1985, p. 6.

VI.8.2 Hebdos et quotidiens

ROYER, Jean, «Les terribles vivantes (Axes et eau)», le Devoir, samedi 9 mars 1985.

\section{VI.9 Kerameikos}

VI.9.1 Périodiques (excluant hebdos et quotidiens)

BIRON, Normand, "Les textures éternelles de la mort", Vie des Arts, vol. XXXIII, $\mathrm{n}^{\circ} 131$, été 1988 , p. 76.

COTNOIR, Louise, «Kerameikos ", Arcade, n 16, octobre 1988; p. 104-105.

DUPRÉ, Louise, *Du geste à la voix. L'immobilité de la mort», Trois, vol. IX, $n^{\circ} 2$, hiver 1987-1988, p. 65-66.

\section{VI.9.10 Hebdos et quotidiens}

ROYER, Jean, *La voix inoubliable de Louky Bersianik ", le Devoir, samedi 5 mars 1988.

TOUPIN, Gilles, «Lumières sur la nécropöle. Lè Kerameikos de Louky Bersianik », la Presse, 28 mai 1988.

\section{VI.10 La Main tranchante du symbole \\ VI.10.1 Périodiques}

AUDET, Élaine, « Naître du mauvais bord de la langue »; l'Aut'Journal, $\mathrm{n}^{\circ} 88$ décembre 1990.

YANACOPOULO, Andrée, * Reprises. La Main tranchante' du symbole [...]», Spirale, n 104, mars 1991, p. 4.

\section{VI.10.2 Quotidien}

VOISARD, Anne-Marie, * Ils en appellent au drame », le Soleil, 6 décembre 1990. 


\section{V1.11 Trace et contraste (disque)}

VI.11.1 Périodiques (excluant hebdos et quotidiens)

GUILBERT, Manon, «Quand on a la passion de l'écriture », le Compositeur canadien, $\mathrm{n}^{\circ} 179$, mars 1983 , p. 25-27.

\section{VI.11.2 Hebdos et quotidiens}

LAVOIE, Denis, «De retour de Spa, Richard Séguin Grand Prix [...] Trace et contraste », la Presse, samedi 18 juillet 1981.

PETROWSKY, Nathalie, «ichard Séguin et Louky Bersianik: Pour une coexistence pacifique: Trace et contraste*, le Devoir, "L'écrivain et le chanteur *, samedi 6 décembre 1980.

\section{Thèses et autres études inédites}

ARBOUR, Kathryn Mary, «French Feminists Re-Visions: Wittig, Rochefort, Bersianik and D'Eaubonne Re-Write Utopia», Dissertation Abstracts International, vol. XLV, $\mathrm{n}^{\circ}$ 2, août 1984, p. 535a.

COISNEL, Anne, «L'à-corps retrouvé(e) - Étude du Pique-nique sur l'Acropole *, M.A., Université de l'Alberta, printemps 1985.

DUVAL, Nathalie, «Étude de la réception générale du Deuxième Sexe de Simone de Beauvoir au Québec francophone et au Canada anglophone», Chap. 3, C: "Louky Bersianik: des prolongements d'une lecture ", M.A., Université Paris X, Nanterré, 1989, p. 89-100.

GRUNDMANN, Erika, «Louky Bersianik, Jeanne Hyvrard et Assia Djebar prennent leur place de sujet dans la langue colonisatrice ", M.A., Université de Victoria, C.-B., 1989.

MARCOTTE, Pierrette, *L'Euguélionne ou la désintégration positive , communication prononcée à la Conférence des Femmes-Écrivains en Amérique, Université d'Ottawa, mai 1978.

OUVRARD, Hélène, * Là littérature féminine québécoise. Une double libération », texte d'une conférence prononcée à l'hôtel Massa; à Paris, le 29 novembre 1977.

RENAULT, Odile Annie, «L'Euguélionne de Louky Bersianik: une écriture au féminin ? », M.A., Université McMaster, Hamilton, juin 1980.

SCOTT, Howard, «Louky Bersianik's l'Euguélionne: Problems of Translating the Critique of Language in New Quebec Feminist Writing ", M.A., université Concordia, Montréal, mai 1984, $157 \mathrm{f}$.

VIAU, Robert, «La folie d'Avertine dans le Pique-nique sur l'Acropole ", dans «Le thème de. la folie dans les romans québécois», Ph.D., Université d'Ottawa.

WEALTI-WALTERS, Jennifer, *The Food of Love: Plato's Banquet and Bersianik's Picnic *, communication au Congrès des Sociétés savantes, Université du Québec à Montréal, Montréal, 1980. 
WEALTI-WALTERS, Jennifer, «Les capitalistes de l'espèce: les hommes dans l'œuvre de Louky Bersianik», communication au Colloque de la NEMLA (Northeast Modern Language), Québec, 1981.

WEALTI-WALTERS, Jennifer, «Louky Bersianik: Women's Identity in a Male Culturew, communication au Colloque de Christchurch, NouvelleZélande, mai 1984.

WEALTI-WALTERS, Jennifer, «Louky Bersianik, fille de Xanthipe », communication au Colloque de Budapest, Hongrie, août 1984. 


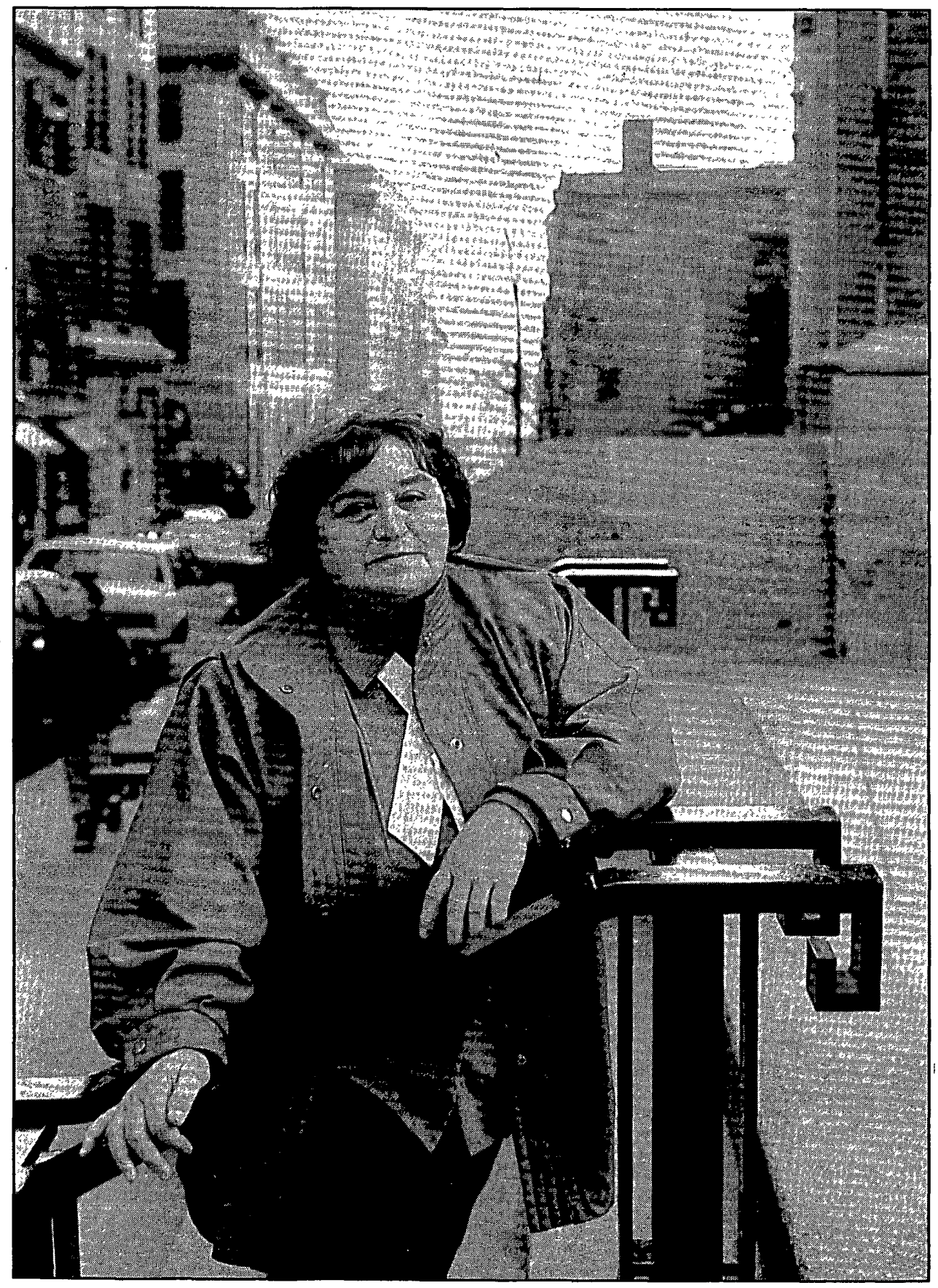

Photo: Josée Lambert 